\title{
Validation of Social Responsiveness Scale for Korean Preschool Children With Autism
}

\author{
Jeeyoung Chun', Guiyoung Bong², Jae Hyun $\mathrm{Han}^{2}$, Miae $\mathrm{Oh}^{3}$, and Hee Jeong Yoo ${ }^{2,4} \bowtie$ \\ ${ }^{1}$ Department of Psychiatry, Seoul National University Hospital, Seoul, Republic of Korea \\ ${ }^{2}$ Department of Psychiatry, Seoul National University Bundang Hospital, Seongnam, Republic of Korea \\ ${ }^{3}$ Department of Psychiatry, Kyung Hee University Hospital, Seoul, Republic of Korea \\ ${ }^{4}$ Seoul National University College of Medicine, Seoul, Republic of Korea
}

Objective This cross-cultural study aims to examine the psychometric properties of the Social Responsiveness Scale (SRS) and to determine the best-estimate-cut-off scores for the diagnosis of autism spectrum disorder (ASD) in Korean preschool children.

Methods A total of 563 children was recruited from multiple sources, including Seoul National University Bundang Hospital. Participants were assessed by a multidisciplinary research team using multiple diagnostic tools based on DSM- 5 diagnostic criteria. Discriminative validity was tested by comparing the difference in SRS scores between ASD and non-ASD groups. Convergent validity was tested by examining correlations between SRS scores with other diagnostic instruments. A receiver operation characteristic curve analysis was conducted to test the sensitivity and specificity of SRS and to determine the best-estimate-cut-off scores for screening ASD in Korean preschool children.

Results There were significant differences in the total SRS scores between the ASD ( $n=242)$ and non-ASD group ( $n=321)$ ( $<<0.01$, 95\% confidence interval [CI]: 14.08-17.24). The differences were significant even after adjusting for age. SRS scores were significantly correlated with other prescreening diagnostic tools for ASD. The best-estimate cut-off score to screen ASD was 55 (area under curve= 0.88 , sensitivity $78.1 \%$, specificity $86.6 \%$ ).

Conclusion The SRS is a valid and reliable instrument to screen and aid in the diagnosis of ASD in Korean preschool children. The adjusted cut-off scores, notably lower than in the original U.S. version, may yield a more accurate diagnosis by reflecting transcultural differences.

Psychiatry Investig 2021;18(9):831-840

Keywords Autism spectrum disorder; Social Responsiveness Scale; Validation; SRS.

\section{INTRODUCTION}

Research places emphasis on the early diagnosis of the autism spectrum disorder (ASD) as the literature consistently highlights the critical role of early intervention in achieving a more positive prognosis. ${ }^{1,2}$ Various screening tools have been proposed and actively employed to promote the earliest possible intervention by measuring the key features of ASD based on Diagnostic and Statistical Manual of Mental Disorders-fifth edition (DSM-5) criteria: persistent and prominent deficits in social in-

Received: May 28, 2021 Accepted: July 5, 2021

$\triangle$ Correspondence: Hee Jeong Yoo, MD, PhD

Department of Psychiatry, Seoul National University Bundang Hospital, Seoul National University College of Medicine, 82 Gumi-ro 173beon-gil, Bundanggu, Seongnam 13620, Republic of Korea

Tel: +82-31-787-7436, Fax: +82-31-787-4058, E-mail: hjyoo@snu.ac.kr

(c) This is an Open Access article distributed under the terms of the Creative Commons Attribution Non-Commercial License (https://creativecommons.org/licenses/bync/4.0) which permits unrestricted non-commercial use, distribution, and reproduction in any medium, provided the original work is properly cited. teraction/communication and restricted and repetitive behaviors (RRBs) and interests, both presenting from an early developmental period. The Social Responsiveness Scale (SRS) is currently one of the most widely used screening tools for ASD worldwide as it has significant advantages.

By examining the traits highly distinct to ASD, the SRS quantitatively measures the continuum of autism symptom severity. ${ }^{3}$ The SRS evaluates the child's social insight, social information processing, mutual social interaction, social anxiety/avoidance, autistic immersion and characteristics. The SRS offers a good interpretation of ASD's genetic/neurologic features, ${ }^{4-6}$ and it has been proven to measure the autistic phenotype transmitted across the generation, ${ }^{7-9}$ which further solidifies its construct validity and utility as a quantitative measure of ASD traits that conveys the intuitive degree of impairment. ${ }^{10}$ As the SRS may be implemented not only by professionally trained practitioners, but also by main caregivers such as parents or teachers, it tends to be less time-consuming and more accessible to chil- 
dren in need as compared to other more formalized diagnostic tools. Another strength of the SRS is that it has been translated and customized into more than 20 official languages and has been employed internationally. ${ }^{11}$

As this screening instrument was originally developed in USA and initially validated with the US population, ${ }^{3,12-14}$ various crosscultural studies have been conducted aiming to confirm the SRS's validity and reliability based on each country's own child population. The SRS has been shown to possess decent psychometric quality when used on large school-based child populations in Germany, ${ }^{15} \mathrm{UK},{ }^{16}$ and Iran. ${ }^{17}$ The SRS has maintained its validity when employed in clinical and school-based samples in Mexico, ${ }^{18}$ mainland China, ${ }^{19}$ Taiwan, ${ }^{20,21}$ Vietnam, ${ }^{22}$ and Japan. ${ }^{23}$ However, majority of cross-cultural validation in literature have been fairly limited to children and the adolescent group between the ages of 4 to 18 years old, as they were the original target of the standard SRS questionnaire. Few studies have confirmed the validity of SRS among adults internationally, ${ }^{24-26}$ and even fewer studies have been conducted focusing mainly on the preschooler/toddler group. ${ }^{21,27}$

In Korea, to the best of authors' knowledge, there exists only a single validation study of the Korean version of SRS (K-SRS) focusing on children aged 6 to 12 years old, which showed that the K-SRS has an adequate degree of reliability and validity among Korean participants. ${ }^{11}$ While the study reflects results from international validation processes, there has been a lack of Korean studies examining the validity of SRS in age groups beyond the standard age group, such as children and adolescents aged 4 to 18 years old. It is necessary to evaluate the validity of the SRS for younger groups such as toddlers, considering the importance of early diagnosis and intervention of ASD.

Furthermore, overestimating ASD traits may potentially result in the wastage of diagnostic/therapeutic resources, while underestimating may result in the failure to make an early diagnosis; both are possible depending on the cut-off score. Literature has repeatedly pointed out that cultural differences may attenuate the original statistical power of diagnostic tools for ASD, especially for Asian populations. ${ }^{21,23}$ Thus, the original SRS cut-off scores recommended by the official U.S. manual may result in less than optimal sensitivity/specificity in the transcultural context, prompting a call for the standardization process and for setting unique cut-off scores for screening based on each cultural/ethnic population. ${ }^{27}$ However, there have been no studies that aim to determine a standardized SRS cut-off score for the population of Korean preschoolers and toddlers.

Given this context, this study aims to examine the psychometric properties of the Korean version of the SRS-2 Preschool form, including the questionnaire's discriminative validity against ASD, other developmental disorders (OD), and the typical development (TD) group among Korean preschool children. Ad- ditionally, we determined the best-estimate-cut-off scores for the screening of ASD in Korean preschool children while also conducting a satellite analysis to examine the correlation between the SRS scores of children with ASD and their parents and perform a preliminary exploration on the inheritable end phenotype of ASD, which is the main target of SRS evaluation.

\section{METHODS}

\section{Participants}

The participants for this study were drawn from the databases of two previous and ongoing studies, including a genetic study regarding ASD's biomarkers and a study on the development of an early ASD screening instrument. ${ }^{28}$ Toddlers and young children between the ages of 10 to 65 months along with their parents were recruited from multiple sources including Seoul National University Bundang Hospital, local primary clinics, and via conventional methods including flyers and referrals from already participating parents. Children with severe medical conditions or neurological difficulties, severe sensory/motor impairments, or those with very low compliance precluding the assessment were excluded during recruitment. The study was approved by the Institutional Review Board at Seoul National University Bundang Hospital (IRB no. B-1911/577-106). Written informed consent were acquired from all parents of children during the collection of original data, and consent was waived for post-hoc analysis.

\section{Procedures}

\section{Measures}

\section{Autism Diagnostic Observation Schedule (ADOS) and} ADOS-2 $2^{29}$

ADOS and ADOS-2 are semi-structured standard diagnostic tools employing a play-based approach to detect the ASD symptoms. ADOS consisted of modules 1 to 4 , while ADOS-2 consisted of module $\mathrm{T}$, and these aimed to evaluate both verbal and nonverbal communication and social interaction as well as the child's playing and imagination. Both modules targeted children below the age of 30 months. The examined child is assessed on different domains related to key symptoms of ASD: communication, social interaction, combined communication and interaction, play and imagination, and RRBs using ADOS; social interaction and communication, RRBs, and total and comparison scores using ADOS-2. Children are classified into three groups based on their final results: autism, ASD, and nonspectrum combined. Participants were assessed using ADOS until ADOS-2 was published in Korea; from then on, ADOS-2 was used. Based on the ADOS-2 algorithms, premeasured 
ADOS scores were rescored into an ADOS-2 score form. Western Psychological Services, the creator of ADOS-2, approved the Korean translation of ADOS-2. ${ }^{30}$

\section{Autism Diagnostic Interview-Revised (ADI-R $)^{31}$}

ADI-R is a semi-structured parent interview tool for parents with children aged 2 or older. Generally, it supplements the direct observation of children using the ADOS assessment. The contents of the interview consist of early development, acquisition and loss of language/other functions, language and communication skills, social development and play, interest and behavior, and general behavior. It evaluates three maladjustment symptoms of ASD: a persistent and prominent deficit in social interaction/communication, RRBs, and restricted and repetitive interests, based on diagnostic algorithms of DSM-IV and the International Statistical Classification of Diseases and Related Health Revisions 10th Revision. ${ }^{32}$ Each question is coded as follows: 0 points for the absence of maladjustment pattern, 1 point for some maladjustment patterns, and 2 points for significant maladjustment patterns. ASD is diagnosed if all scores in the three behavioral areas exceed the cut-off scores. The cutoff scores are 8 points for communication (7 points in cases of communication difficulties), 10 points for social interaction, and 3 points for RRBs and interests. ADI-R was administered to participants aged younger than 2 in this study, which included a diagnostic impression and detailed history taking, though this tool was originally intended for children over the mental age of 2 years old. Western Psychological Services approved the Korean translation of the ADI-R. ${ }^{33}$

\section{Korean version of Childhood Autism Rating Scale (K-CARS) $)^{34}$}

K-CARS detects and measures the severity of pervasive developmental disorder symptoms based on both the parents' report and direct observation of children. K-CARS consists of 15 items evaluating different domains including socialization, language and nonverbal communication, restricted emotional responses or unusual behaviors and interests, and sensory sensitivities. Each item is scored from 1 (no impairment) to 4 (severe impairment). Upon the completion of the assessment, the child is categorized as having either mild, moderate, or severe symptoms of autism. We used a cut-off score of 24 to diagnose ASD based on the previous standardization process..$^{35,36}$

\section{Korean Social Communication Questionnaire (K-SCQ) ${ }^{37}$}

Based on the ADI-R, the Social Communication Questionnaire (SCQ) is a screening tool consisting of 40 questions that ask parents or caregivers about ASD-related symptoms (communication, mutual social interaction, RRBs and interests). There are two types: "Lifetime Form" and "Current Form." Lifetime Form is based on the overall development of the child and
Current Form is based on the child's behavior in the last three months. Each question can be answered with a "yes" or "no," while the first question checks the child's ability to speak more than two words. If the first question is answered with "Yes," the total score is the sum of the scores of questions 2 to 40 . If the first question is answered with "No," the total score is the sum of the scores of questions 8 to 40 . We used a cut-off score of 10 to diagnose a high risk of ASD based on a standardization study previously conducted in Korea. ${ }^{38}$ Translation and backtranslation were conducted for the publication of the Korean version of SCQ, and the completed version was published after the original author's review of the back-translated version and two more revision processes.

\section{Social Response Scale Second Edition (SRS-2) $)^{14}$}

\section{SRS-2 Preschool Form}

With the approval of the original publisher, Western Psychological Services, we translated and back-translated the SRS-2 preschool form and received permission to use it for the study. The SRS preschool form consists of 65 questions that ask parents or caregivers, including teachers, about the characteristics of the child's social interactions based on their behavior for the past six months. Each question is answered on a scale ranging from "Not at all" (0 points) to "Almost always" ( 3 points), with a total score of 195 . The higher the score, the lower the social dexterity.

The original SRS-2 preschool version was intended for children above the age of 30 months. However, as a substantial number of participants aged less than 30 months were recruited, we planned to conduct a subgroup analysis for children over 30 months and for those below 30 months to determine the validity of SRS in the age group below the original stipulation and to prevent potential bias from lenient age inclusion. In this study, the SRS- 2 was filled out by a primary caregiver who was familiar with the developmental process of the child. The SRS's testretest reliability coefficient of the U.S. preschool version was $0.75 ;^{3,39}$ the Cronbach's $\alpha$ was 0.98 and 0.96 based on a previous study involving the Korean school age population. ${ }^{28,38}$ Cronbach's $\alpha$ of the SRS-2 preschool form used in this study was 0.96 .

\section{SRS-2 Adult Self-Report form}

The SRS-2 adult self-report form (the SRS-2 ASR) is indicated for examinees aged 19 through 89. It consists of 65 questions scored on a Likert scale identical to the preschool form, with each item being scored from $0-3$ points.

Vineland Adaptive Behavior Scales, Second edition (VABS-2) ${ }^{40}$

The VABS-2 was designed to assess the adaptive behavior and individual and social skills necessary for independent dai- 
ly living over a lifetime, and is conducted through a survey interview and evaluation from parents and caregivers, including teachers. It aims to assess the child's communication, daily living skills, social skills, motor skills, as well as maladaptive behaviors which can be both internal and external. Items are rated from 0 to 2 with 0 indicating that a certain skill is not used by the examinee at all; 1 indicating that the skill is used infrequently; and 2 indicating that the skill is used frequently. VABS- 2 can also be used to screen the service eligibility, to plan additional interventions, and to track and review the changes in children with ASD post-intervention.

Sequenced Language Scale for Infants. Sequenced Language Scale for Infants (SELSI) ${ }^{41}$

SELSI evaluates the receptive language development and expressive language for children between the ages of 5 to 36 months. Consisting of 56 questions, SELSI provides the developmental age, language development in terms of percentile and standard deviation, and the approximate level of development for children with delays.

\section{Diagnostic procedures}

Recruited children were assessed using the aforementioned diagnostic instruments. Parents were evaluated using the SRS-2 adult self-report form. Basic demographics were obtained from all participants. Developmental history and family history were extensively surveyed for diagnostic aid. Qualified diagnostic assessment staff, education professionals specializing in ASD evaluation, and experienced clinicians, including two licensed child psychiatrists, made the final clinical best estimate diagnoses. Any uncertain results were discussed using a multidisciplinary team approach at weekly meetings, where the videotaped evaluations were discussed and reviewed to draw conclusions. We classified the children into three diagnostic groups; the participant group with ASD included children with a final clinical best-estimate diagnosis of ASD based on all their exam results and information, following the DSM- 5 criteria. As for children aged 18-23 months, while this age is sometimes considered too young for confirming a diagnosis, we diagnosed the child with ASD if they were assessed to have "mild-to-moderate concerns" or "moderate-to-severe concerns" in module T of ADOS-2, and when their parents reported concurrent and equivalent degrees of concerns in ADI-R. The OD group included children whose levels of development were below -1 standard deviation in SELSI, or those who scored less than 70 points in any domain of VABS. The TD group included children showing a normal range of development, and those who did not meet the criteria for the diagnosis of either ASD or TD.

\section{Statistical analyses}

The discriminative validity of SRS was tested by conducting a t-test and analysis of covariance to compare differences in scores between ASD and non-ASD groups. The convergent validity of SRS with other diagnostic instruments including KCARS and K-SCQ was examined using Pearson's correlation. Finally, we conducted a receiver operation characteristic (ROC) curve analysis to test the sensitivity and specificity of SRS, and to determine the best-estimate-cut-off scores for screening ASD in Korean preschool children. An area under curve (AUC) serves as the indicator of performance, and a value of 0.50 means that the diagnostic tool has no discriminative power as compared to the gold standard, while a value of 1.0 means complete power. A value of 0.70 or higher indicates an appropriate level of discriminative accuracy. Sensitivity, specificity, positive predictive values, and negative predictive values were calculated. We used MedCalc for Windows, version 20 (MedCalc Software, Ostend, Belgium) for a ROC curve analysis and SPSS statistics 25 and Subscription (IBM Corp., Armonk, NY, USA) for other analyses. The confidence interval was set at $95 \%$.

\section{RESULTS}

\section{Participant characteristics}

A total of 563 children ranging from 10 to 65 months of age participated in the study. The average age was 31.47 months $(\mathrm{SD}=9.95)$, and the majority of participants were male $(\mathrm{n}=379$, $67.32 \%$ ). A total of 335 children aged 30 months and above were recruited $(n=335$, mean age $=38.16, S D=6.14)$, while 228 children aged below 30 months were recruited $(n=228$, mean age $=$ 21.67, $\mathrm{SD}=5.16$ ).

Participants were classified according to the clinical best estimate diagnosis into three groups: ASD ( $n=242), \mathrm{OD}(\mathrm{n}=74)$, and TD ( $n=247)$. Upon combining the OD and TD into the non-ASD group, there were 321 participants. The mean age of the ASD group was 35.64 months $(\mathrm{SD}=9.27)$, higher than the OD group (mean age $=29.66, \mathrm{SD}=8.03$ ) and TD group (mean age=27.93, $\mathrm{SD}=9.6$ ). The mean VABS score was lowest in the ASD group (mean=69.28, $\mathrm{SD}=14.29$ ), followed by the OD group (mean=81.26, $\mathrm{SD}=11.74$ ), and the TD group (mean=101.04, $\mathrm{SD}=12.80$ ). K-CARS scores, on the other hand, were lowest in the TD group (mean=16.65, $\mathrm{SD}=1.94$ ), and higher in the OD group (mean=20.02, $\mathrm{SD}=4.37$ ) and the ASD group (mean= 31.44, $\mathrm{SD}=5.08$ ), displaying a similar pattern with their SCQ scores $(\mathrm{TD}$ mean=6.20, $\mathrm{SD}=4.03$; $\mathrm{OD}$ mean $=8.01, \mathrm{SD}=4.58$; $\mathrm{ASD}$ mean=15.47, $\mathrm{SD}=6.38$ ).

The demographic characteristics of the participants, along with the participants' assessment scores other than SRS, are summarized in Table 1. For the satellite study, a total of 548 adults were recruited from the pool of parents of participants, 
consisting of 272 fathers and 276 mothers.

\section{Discriminative validity}

Overall, the mean SRS score of the ASD group was 63.26 with an SD of 10.73, whereas the mean score of the non-ASD group was 47.60 , with an SD of 7.46, indicating a statistically significant difference $(\mathrm{t}=19.44, \mathrm{p}<0.01)$. Statistical significance was maintained after adjusting for age $(\mathrm{F}=362.84, \mathrm{p}<0.01)$. When we restricted the participants according to the original indication age of SRS preschool form (children aged 30 months and above), the mean score of the ASD group was $64.39(\mathrm{SD}=10.12)$ and the mean score of the non-ASD group was $46.75(\mathrm{SD}=8.28)$, still showing a statistical difference $(\mathrm{t}=17.55, \mathrm{p}<0.01)$ with and without adjusting for age $(\mathrm{F}=289.21, \mathrm{p}<0.01)$. As for participants below the age of 30 months, there was still a significant difference between the ASD and non-ASD groups, with and without adjusting for age (Table 2).

Upon classifying all the participants into three groups (ASD, $\mathrm{OD}$, and TD), the mean score of the OD group was 52.01 ( $\mathrm{SD}=$ 8.07 ) and the mean score of the TD group was $46.28(\mathrm{SD}=6.74)$, with a statistically significant difference among all three groups $(\mathrm{F}=147.05, \mathrm{p}<0.01)$. Among the three groups, the score was highest for the ASD group, followed by OD, and lastly, the TD group. The significance was maintained after adjusting for age

Table 1. Demographic result

\begin{tabular}{|c|c|c|c|c|c|}
\hline & $\mathrm{N}$ & Mean age $\pm S D$ & $\mathrm{VABS}$ mean $\pm \mathrm{SD}$ & $\mathrm{K}-\mathrm{CARS}$ mean $\pm \mathrm{SD}$ & SCQ mean \pm SD \\
\hline Total & 563 & $31.47 \pm 9.95$ & $84.87 \pm 19.94$ & $23.35 \pm 8.01$ & $10.33 \pm 6.82$ \\
\hline \multicolumn{6}{|l|}{ Sex } \\
\hline Male & 379 & $32.43 \pm 9.76$ & $82.03 \pm 19.27$ & $24.36 \pm 7.95$ & $11.18 \pm 6.72$ \\
\hline Female & 184 & $29.51 \pm 10.07$ & $90.73 \pm 20.08$ & $21.30 \pm 7.77$ & $8.64 \pm 6.71$ \\
\hline \multicolumn{6}{|l|}{ Age } \\
\hline$\geq 30$ months & 335 & $38.16 \pm 6.14$ & $79.54 \pm 20.18$ & $25.36 \pm 8.27$ & $11.14 \pm 7.33$ \\
\hline$<30$ months & 228 & $21.67 \pm 5.16$ & $92.63 \pm 16.82$ & $20.52 \pm 6.70$ & $9.18 \pm 5.82$ \\
\hline \multicolumn{6}{|l|}{ Diagnosis } \\
\hline ASD & 242 & $35.64 \pm 9.27$ & $69.28 \pm 14.29$ & $31.44 \pm 5.08$ & $15.47 \pm 6.38$ \\
\hline Non-ASD & 321 & $28.33 \pm 9.28$ & $96.41 \pm 15.09$ & $17.44 \pm 3.06$ & $6.63 \pm 4.23$ \\
\hline \multicolumn{6}{|c|}{ Diagnosis in 3 group } \\
\hline ASD & 242 & $35.64 \pm 9.27$ & $69.28 \pm 14.29$ & $31.44 \pm 5.08$ & $15.47 \pm 6.38$ \\
\hline DD & 74 & $29.66 \pm 8.03$ & $81.26 \pm 11.74$ & $20.02 \pm 4.37$ & $8.01 \pm 4.58$ \\
\hline $\mathrm{TD}$ & 247 & $27.93 \pm 9.60$ & $101.04 \pm 12.80$ & $16.65 \pm 1.94$ & $6.20 \pm 4.03$ \\
\hline
\end{tabular}

ASD, autism spectrum disorders; DD, developmental disorder; TD, typical development; SD, standard deviation; VABS, Vineland Adaptive Behavior Scales; K-CARS, The Korean version of Childhood Autism Rating Scale; SCQ, Social Communication Questionnaire

Table 2. Mean differences in SRS score between ASD, non-ASD (OD+TD)

\begin{tabular}{|c|c|c|c|c|c|c|c|c|}
\hline & \multirow[t]{2}{*}{$\mathrm{N}$} & \multirow[t]{2}{*}{ Mean \pm SD } & \multirow{2}{*}{$\begin{array}{c}\text { Standard } \\
\text { error of the } \\
\text { means }\end{array}$} & \multirow[t]{2}{*}{$\mathrm{t}$-value } & \multirow{2}{*}{$\mathrm{p}$} & \multirow[t]{2}{*}{$95 \% \mathrm{CI}$} & \multicolumn{2}{|c|}{$\begin{array}{l}\text { Mean differences } \\
\text { adjusted by the age }\end{array}$} \\
\hline & & & & & & & F-value & $\mathrm{p}$ \\
\hline Total & 563 & $54.33 \pm 11.88$ & & & & & & \\
\hline Diagnosis & & & & 19.44 & $<0.01^{*}$ & $14.08-17.24$ & 362.84 & $<0.01^{*}$ \\
\hline ASD & 242 & $63.26 \pm 10.73$ & 0.69 & & & & & \\
\hline Non-ASD & 321 & $47.60 \pm 7.46$ & 0.42 & & & & & \\
\hline Over 30 months & & & & 17.55 & $<0.01^{*}$ & $15.66-19.62$ & 289.21 & $<0.01^{*}$ \\
\hline ASD & 185 & $64.39 \pm 10.12$ & 0.74 & & & & & \\
\hline Non-ASD & 150 & $46.75 \pm 8.28$ & 0.68 & & & & & \\
\hline Below 30 months & & & & 6.8 & $<0.01^{*}$ & $7.93-14.51$ & 79.37 & $<0.01^{*}$ \\
\hline ASD & 57 & $59.56 \pm 11.86$ & 1.57 & & & & & \\
\hline Non-ASD & 171 & $48.34 \pm 6.59$ & 0.50 & & & & & \\
\hline
\end{tabular}

Mean differences compared with independent $\mathrm{t}$-test, ${ }^{*} \mathrm{p}<0.05$. Age adjusted with analysis of covariance, ${ }^{*} \mathrm{p}<0.05$. ASD, autism spectrum disorders; OD, other developmental disorder; TD, typical development; SD, standard deviation; CI, confidence interval 
$(\mathrm{F}=147.05, \mathrm{p}<0.01)$. In the subgroup analysis of children aged above 30 months, the mean score of OD was 50.98 ( $\mathrm{SD}=8.82$ ) and the mean score of TD was $45.22(\mathrm{SD}=7.54)$, and maintained a significant difference $(\mathrm{F}=158.16, \mathrm{p}<0.01)$ within the three groups, even after adjusting for age $(\mathrm{F}=132.67, \mathrm{p}<0.01)$. Again, there was a significant difference among the three groups in terms of SRS mean scores for children below the age of 30 months, with and without the age adjustments (Table 3 ).

\section{Comparison with SCQ \& K-CARS}

We analyzed the correlations between SRS, SCQ and K-CARS. There was a significant correlation between SRS scores and SCQ scores $(\mathrm{r}=0.800, \mathrm{p}<0.01)$ and between SRS scores and K-CARS scores $(r=0.730, p<0.01)$ for all the participants. The positive correlation was maintained after adjusting for age $(r=0.802$, $\mathrm{p}<0.01$ in SRS \& SCQ; $\mathrm{r}=0.711, \mathrm{p}<0.01$ in SRS \& K-CARS). When we restricted the analysis to include only children above the age of 30 months, a stronger correlation was established between the measures $(r=0.893, p<0.01$ in SRS \& SCQ; $r=0.737$, $\mathrm{p}<0.01$ in SRS \& K-CARS). The correlation became weaker but still remained significant when we restricted the analysis to include only children below the age of 30 months ( $r=0.695, \mathrm{p}<$ 0.01 in SRS \& SCQ; $r=0.657, p<0.01$ in SRS \& K-CARS).

\section{Correlation with parents' SRS score}

Upon analyzing the correlation between the SRS scores of all the children and those of their parents, there was a weak corre-

Table 3. Mean differences in SRS score between ASD, OD \& TD

\begin{tabular}{|c|c|c|c|c|c|c|}
\hline & \multirow[t]{2}{*}{$\mathrm{N}$} & \multirow[t]{2}{*}{ Mean \pm SD } & \multirow[t]{2}{*}{$\mathrm{F}$} & \multirow[t]{2}{*}{$\mathrm{p}$} & \multicolumn{2}{|c|}{$\begin{array}{c}\text { Mean differences } \\
\text { adjusted } \\
\text { by the age }\end{array}$} \\
\hline & & & & & F-value & $\mathrm{p}$ \\
\hline Total & 563 & $54.33 \pm 11.88$ & & & & \\
\hline Diagnosis & & & 229.08 & $<0.01^{*}$ & 147.05 & $<0.01^{*}$ \\
\hline ASD & 242 & $63.26 \pm 10.73$ & & & & \\
\hline OD & 74 & $52.01 \pm 8.07$ & & & & \\
\hline TD & 247 & $46.28 \pm 6.74$ & & & & \\
\hline \multicolumn{2}{|c|}{ Over 30 months } & & 158.16 & $<0.01^{*}$ & 132.67 & $<0.01^{*}$ \\
\hline ASD & 185 & $64.39 \pm 10.12$ & & & & \\
\hline OD & 40 & $50.98 \pm 8.82$ & & & & \\
\hline $\mathrm{TD}$ & 110 & $45.22 \pm 7.54$ & & & & \\
\hline \multicolumn{2}{|c|}{ Below 30 months } & & 50.61 & $<0.01^{*}$ & 21.95 & $<0.01^{*}$ \\
\hline ASD & 57 & $59.56 \pm 11.86$ & & & & \\
\hline OD & 34 & $53.24 \pm 7.07$ & & & & \\
\hline $\mathrm{TD}$ & 137 & $47.12 \pm 5.90$ & & & & \\
\hline
\end{tabular}

Mean differences compared with analysis of variance, ${ }^{*} \mathrm{p}<0.05$. Age adjusted with analysis of covariance, ${ }^{*} \mathrm{p}<0.05$. ASD, autism spectrum disorders; OD, other developmental disorder; TD, typical development; SD, standard deviation; CI, confidence interval lation between the scores of children and their mothers ( $\mathrm{r}=$ $0.175, \mathrm{p}<0.01$ ). There was no significant correlation between the SRS scores of children above 30 months old and those of their parents. Still, there was a weak significant correlation between the SRS scores of children below 30 months old and their mothers $(r=0.236, p<0.05)$. There were no significant correlations between the SRS scores of children and that of their fathers in both age groups. There was no significant correlation between the SRS scores in children diagnosed with ASD and those of their parents, while the scores of children without ASD had a weak yet statistically significant correlation with those of both of their parents (mother-non-ASD child: $r=0.35$; fathernon-ASD child: $r=0.24$; both $\mathrm{p}<0.01$ ).

\section{ROC curve analysis}

We conducted a receiver operating characteristic (ROC) analysis to generate the optimal cut-off score to diagnose ASD in SRS. Upon analysis of the data of all participants, SRS showed solid diagnostic accuracy with an AUC of 0.883 . When we apply the cut-off score of 55.50, the test showed optimal results with a sensitivity of $78.1 \%$ and specificity of $86.6 \%$ (Figure 1 ).

In group of children aged above 30 months, SRS still maintained its strong diagnostic accuracy with an AUC of 0.904 . The optimal cut-off score was 55 , with a sensitivity of $82.7 \%$ and specificity of $86.0 \%$ (Figure 2). In children aged below 30 months, AUC was 0.803 and the optimal cut-off score was 51, with a sensitivity of $80.7 \%$ and specificity of $70.8 \%$ (Figure 3 ).

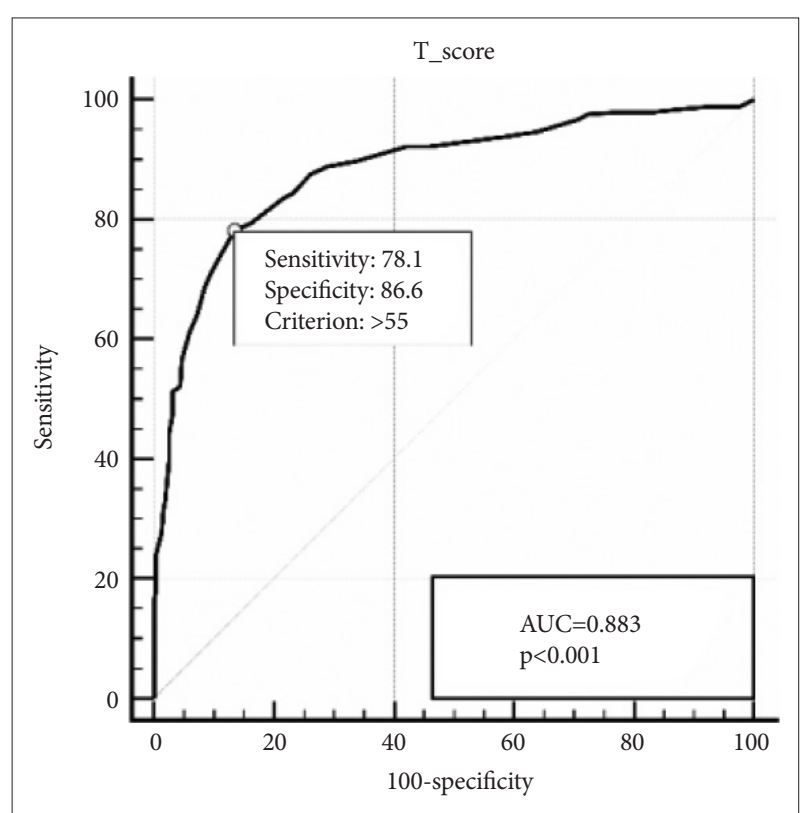

Figure 1. Receiver operating characteristic curve of the SRS for ASD vs. non-ASD. SRS, Social Responsiveness Scale; ASD, autism spectrum disorders; AUC, area under the curve. 


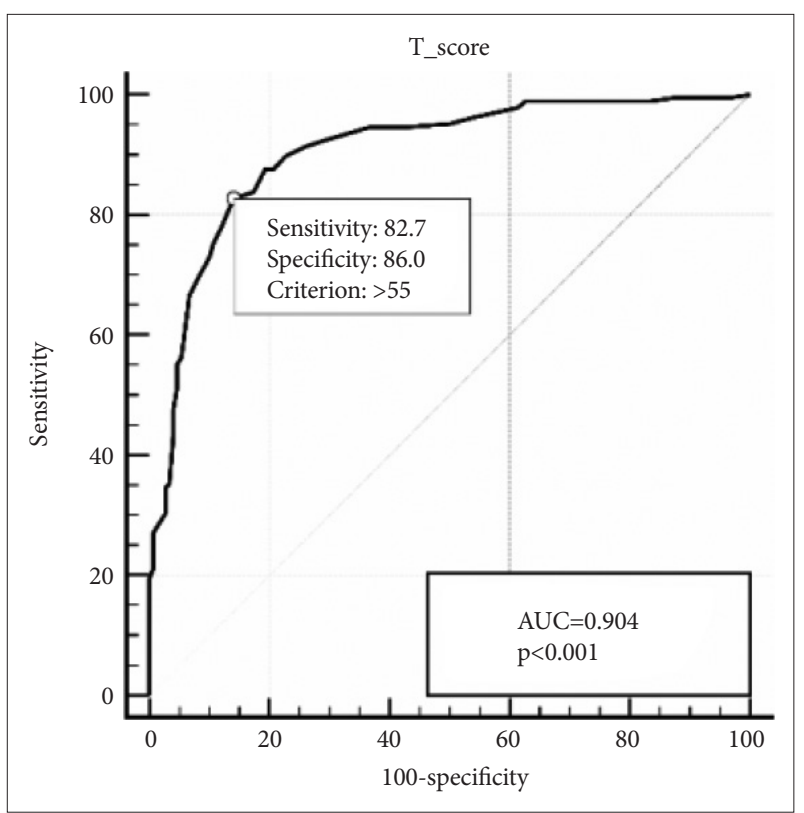

Figure 2. ROC Curve of the SRS for ASD vs. non-ASD for children aged 30 months and older. ROC, receiver operating characteristic; SRS, Social Responsiveness Scale; ASD, autism spectrum disorders; AUC, area under the curve.

\section{DISCUSSION}

This study is the first to establish the validity of the SRS preschool form when used on Korean children. The mean scores of the SRS were statistically different between the ASD and nonASD group, and these also significantly differed among the ASD, $\mathrm{OD}$, and TD groups. Adjusting for age did not affect the significance. This may suggest that SRS may be employed not only as a screening instrument to differentiate ASD and non-ASD, but may also aid in the in-depth screening and diagnosis of ASD, supporting the endeavor to detect and differentiate ASD from other development disorders. Another study by the author has also demonstrated the high validity of the Korean ADI-R in ASD detection in comparison with the OD group. ${ }^{42}$

Furthermore, the significant difference in SRS mean scores was maintained in the subgroup analysis of children aged below 30 months. The result supports that the SRS preschool form may sustain its diagnostic efficacy in Korean children population even below its original indicated age group, and can thus aid in prescreening procedures for children below 30 months. Considering the importance of making the earliest possible diagnosis and providing intervention in the course of managing ASD, ${ }^{43,44}$ being able to apply the SRS in an even younger age group may yield great implications for clinical practice.

Our results correspond with literature based on the East Asian preschool population. Stickley et al. ${ }^{27}$ employed the Japanese preschool version of the SRS (SRS-P), ${ }^{39}$ which is a modified variation of the original SRS and almost identical to the latest

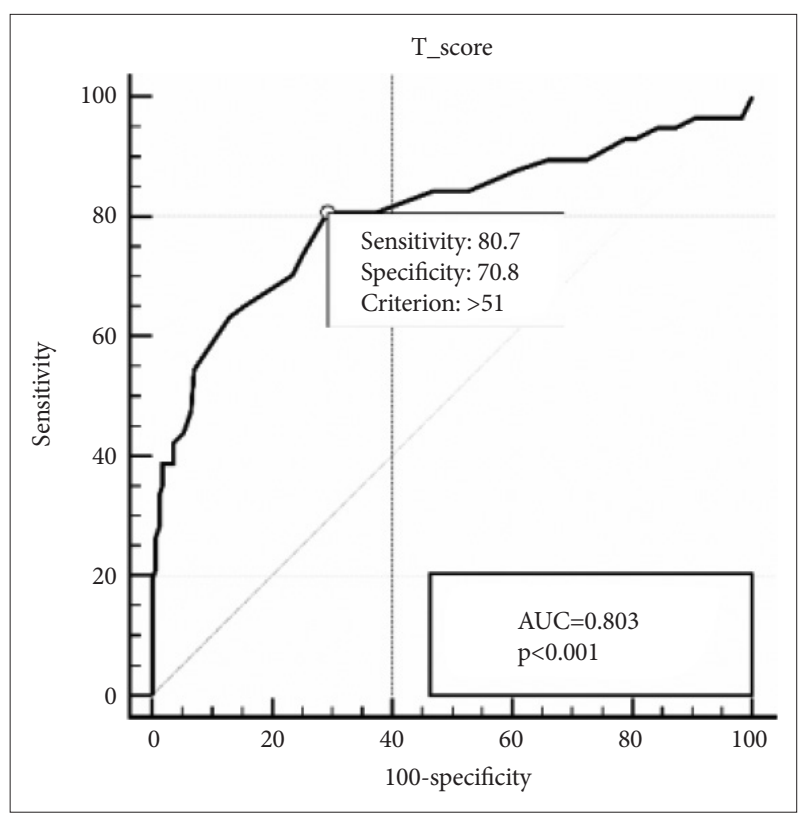

Figure 3. ROC Curve of the SRS for ASD vs. non-ASD for children aged 30 months. ROC, receiver operating characteristic; SRS, Social Responsiveness Scale; ASD, autism spectrum disorders; AUC, area under the curve.

SRS-2 Preschool Form intended for children aged between 30 to 54 months. ${ }^{14}$ In the study, which included a clinical and community based sample of toddlers between the age of 26 to 51 months in Japan showed a good degree of reliability and validity in terms of SRS-P scores, proving SRS-P's utility in the process of screening and diagnosing ASD in Japanese preschool children. Our subgroup analysis of children aged below 30 months further validates Stickley et al's ${ }^{27}$ supposition that the autistic end phenotype can be discernible and quantitatively measured at an even earlier age than 2 to 4.5 years old. In an earlier work, Wang et al. ${ }^{21}$ employed the original SRS to evaluate a clinical and community based sample of Taiwanese children aged 4 to 6 , validating its solid psychometric property to diagnose ASD.

We verified the convergent validity of SRS by identifying relevant correlations of SRS with SCQ and K-CARS. There were positive and significant correlations between SRS and SCQ, and SRS and K-CARS across all age groups, but this correlation was strongest among children aged above 30 months, which was the age group for which the SRS preschool form was originally designed.

According to our analysis, the optimal cut-off score of SRS was 55 for the entire age group, which was substantially lower than the original U.S. cut-off score of 60 which is also currently being employed in the Korean clinical field. The findings support the previous study on the use of SRS on the Korean school age population which found much lower optimal cut-off scores, 52 for males and 51 for females, compared to the U.S. cut-off 
scores of 70 for males and 65 for females. ${ }^{11}$ Based on our results, the direct application of the U.S. cut off score will result in a higher false-negative rate among Korean children, thus deterring early accurate screening and appropriate intervention.

In addition, as SRS is a diagnostic measure which mainly depends on the self-reports of the main caregivers, the difference in the optimal cut-off score may reflect cultural differences or even differing health literacy levels among the caregivers in each country, featuring a wide spectrum of perspectives in the evaluation and classification of children's behavior as typical or autistic based on their cultural background. The literature has shown consistent differences between the original and Korean cut-off scores in other ASD diagnostic tools. For example, the original cut-off score of K-CARS was 30, but the optimal cutoff score based on Korean data was 28.5 for all participants (sensitivity=0.871, specificity=0.8), and 24.5 for the high-functioning ASD group (sensitivity $=0.759$, specificity $=0.771$ ). ${ }^{36}$ The optimal cut-off score for the SCQ was also lower for Korean children. Compared to the original cut-off score of 15, the optimal cut-off scores were 12 points for children aged 48 months and above, and 10 points for children below 47 months based on Korean data. ${ }^{38}$ The consistent lower cut-off scores in Korean data across various screening tools which depend on self-reports may indicate relatively lower recognition of the symptoms or risk signs of $\mathrm{ASD},{ }^{42}$ or the tendency of Korean caregivers to report the children's status in a more positive manner and with more caution due to the negative social stigma surrounding behavioral and mental health problems; ${ }^{11}$ conversely, they may have a more lenient standards in evaluating the children's behavior. The findings suggest that the risk of false negative diagnoses is even higher in the Korean children population since the diagnosis is made solely based on the caregivers' self-reports. A comprehensive evaluation by professionals may have greater implications in this matter.

The SRS has been employed as a surrogate to examine and quantify the relationship of autistic traits within the family in past studies. Constantino and $\operatorname{Todd}^{7}$ showed statistically significant intraclass correlation (ICC) between the SRS scores of children and their parents (mother-daughter $=0.41$, motherson $=0.38$, father - daughter $=0.49$, father - son $=0.58$ ). We also conducted the satellite analysis exploring the correlation between the SRS scores of children and their parents. There was a significant positive correlation between the SRS scores of children and their mothers overall and in the group aged 30 months and below.

However, when we confined the analysis to children with ASD, their scores had no significant correlation with those of their parents. In contrast, there was a weak but significant correlation between the scores of children without ASD with those of both their parents. These results replicate the pattern observed in previous studies. A study by Lyall et al. ${ }^{8}$ demonstrated that the parental autistic traits measured through SRS scores can potentially predict the risk of the diagnosis of ASD in their children; however, there was no direct correlation between the SRS scores of children with ASD and their parents (mother-ASD child: $r=0.02, \mathrm{ICC}=0.0$; father $-\mathrm{ASD}$ child: $\mathrm{r}=0.13, \mathrm{ICC}=0.0$ ), while there was a moderate degree of correlation between the scores of children without ASD and their parents (mother-nonASD child: $r=0.30$, ICC $=0.31$; father-non-ASD child: $r=0.42$, ICC $=0.33$ ). The lack of a significant correlation between the scores of children with ASD and their parents was further supported by a study conducted by Page et al, ${ }^{9}$ who also found a strong correlation between the scores of children without ASD and their parents (mother-ASD child: $\mathrm{ICC}=0.11$; father-ASD child: ICC $=0.27$; mother - non-ASD child: $\mathrm{ICC}=0.65$; father non-ASD child: $\mathrm{ICC}=0.68$ ).

The absence of a correlation between the SRS scores of children with ASD and those of their parents has been postulated to be due to the restriction in score ranges in children with ASD compared to children without ASD, ${ }^{8}$ and further studies are necessary to exclude if there is an additional transcultural impact of parents' evaluation on themselves. Similar to the case in children, parents may under-recognize or underreport their status due to various cultural norms/backgrounds. Our result, overall, supports the hypothesis that there is a significant correlation between the observable autistic traits in parents and their children which could be more prominently measured in the subsyndromal range across the normal population, and those end phenotypes may be the ramification of genetic inheritability. ${ }^{8,45}$

The study has several limitations. First, multiple evaluators conducted the assessment procedures resulting in uncertain inter-rater reliability, which may reduce the accuracy and coherent validity of the entire data. Secondly, there was a relatively small number of participants aged below 30 months with ASD, which may further reduce the statistical validity in the relative subgroup analysis. The optimal cut-off scores in the age group below 30 months notably differed from the other two groups, and had the lowest AUC, sensitivity, and specificity. Lastly, the adult sample was relatively small as well, almost half of the child participants, which may further reduce the statistical power of our satellite study.

In spite of the limitations above, this study provided a rational basis to employ the SRS preschool form for screening and diagnosing ASD among the population of Korean children. The SRS preschool form may also sustain its efficacy under the originally intended age group. Furthermore, the study's adjusted cut-off scores are much lower than the original scores. The application of these customized cut-off scores, which reflect the transcultural difference, would further increase the diagnos- 
tic power of the SRS in the course of ASD evaluation.

\section{Availability of Data and Material}

The datasets generated or analyzed during the study are not publicly available due to the fact that the data are collected from the minors and are subject to IRB review, but are available from the corresponding author on reasonable request.

\section{Conflicts of Interest}

The authors have no potential conflicts of interest to disclose.

\section{Author Contributions}

Conceptualization: Jeeyoung Chun, Guiyoung Bong, Hee Jeong Yoo. Data curation: Jeeyoung Chun, Guiyoung Bong, Hee Jeong Yoo. Formal analysis: Jeeyoung Chun, Guiyoung Bong, Hee Jeong Yoo. Funding acquisition: Hee Jeong Yoo. Investigation: Jeeyoung Chun. Methodology: Jeeyoung Chun, Hee Jeong Yoo. Project administration: Hee Jeong Yoo. Resources: Hee Jeong Yoo. Software: Jeeyoung Chun. Supervision: Hee Jeong Yoo. Validation: Hee Jeong Yoo. Visualization: Jeeyoung Chun. Writingoriginal draft: Jeeyoung Chun. Writing_review \& editing: Guiyoung Bong, Jae Hyun Han, Miae Oh, Hee Jeong Yoo.

\section{ORCID iDs}

$\begin{array}{ll}\text { Jeeyoung Chun } & \text { https://orcid.org/0000-0001-5160-8700 } \\ \text { Guiyoung Bong } & \text { https://orcid.org/0000-0001-8630-9399 } \\ \text { Jae Hyun Han } & \text { https://orcid.org/0000-0003-3994-3463 } \\ \text { Miae Oh } & \text { https://orcid.org/0000-0002-1927-3637 } \\ \text { Hee Jeong Yoo } & \text { https://orcid.org/0000-0003-0521-2718 }\end{array}$

\section{Funding Statement}

This work has been supported by the Korea Health Industry Development Institute, Social Service R\&D project (HI14C2719), and by the Institute of Information \& communications Technology Planning \& evaluation (IITP) grant funded by the Korea government (MSIT) (No. 2019-0-00330, Development of AI Technology for Early Screening of Infant/Child Autism Spectrum Disorders based on Cognition of the Psychological Behavior and Response).

\section{Acknowledgments}

The authors thank Division of Statistics in Medical Research Collaborating Center at Seoul National University Bundang Hospital for statistical analyses.

\section{REFERENCES}

1. Bradshaw J, Steiner AM, Gengoux G, Koegel LK. Feasibility and effectiveness of very early intervention for infants at-risk for autism spectrum disorder: a systematic review. J Autism Dev Disord 2015;45:778794.

2. Estes A, Munson J, Rogers SJ, Greenson J, Winter J, Dawson G. Longterm outcomes of early intervention in 6-year-old children with autism spectrum disorder. J Am Acad Child Adolesc Psychiatry 2015;54:580587.

3. Constantino JN, Davis SA, Todd RD, Schindler MK, Gross MM, Brophy SL, et al. Validation of a brief quantitative measure of autistic traits: comparison of the social responsiveness scale with the autism diagnostic interview-revised. J Autism Dev Disord 2003;33:427-433.

4. Blanken LM, Mous SE, Ghassabian A, Muetzel RL, Schoemaker NK, El Marroun H, et al. Cortical morphology in 6- to 10-year old children with autistic traits: a population-based neuroimaging study. Am J Psychiatry 2015;172:479-486.

5. Duvall JA, Lu A, Cantor RM, Todd RD, Constantino JN, Geschwind $\mathrm{DH}$. A quantitative trait locus analysis of social responsiveness in multiplex autism families. Am J Psychiatry 2007;164:656-662.
6. Lowe JK, Werling DM, Constantino JN, Cantor RM, Geschwind DH. Social responsiveness, an autism endophenotype: genomewide significant linkage to two regions on chromosome 8. Am J Psychiatry 2015; 172:266-275.

7. Constantino JN, Todd RD. Intergenerational transmission of subthreshold autistic traits in the general population. Biol Psychiatry 2005; 57:655-660.

8. Lyall K, Constantino JN, Weisskopf MG, Roberts AL, Ascherio A, Santangelo SL. Parental social responsiveness and risk of autism spectrum disorder in offspring. JAMA Psychiatry 2014;71:936-942.

9. Page J, Constantino JN, Zambrana K, Martin E, Tunc I, Zhang Y, et al. Quantitative autistic trait measurements index background genetic risk for ASD in Hispanic families. Mol Autism 2016;7:39.

10. Lyall K, Hosseini M, Ladd-Acosta C, Ning X, Catellier D, Constantino $\mathrm{JN}$, et al. Distributional properties and criterion validity of a shortened version of the social responsiveness scale: results from the ECHO program and implications for social communication research. J Autism Dev Disord 2021;51:2241-2253.

11. Cheon KA, Park JI, Koh YJ, Song J, Hong HJ, Kim YK, et al. The social responsiveness scale in relation to DSM IV and DSM5 ASD in Korean children. Autism Res 2016;9:970-980.

12. Constantino JN. The Social Responsiveness Scale. Los Angels, CA: Western Psychological Services; 2002.

13. Constantino JN, Gruber CP. Social Responsive Scale: Manual. Los Angeles, CA: Western Psychological Services; 2005.

14. Constantino JN, Gruber CP. Social Responsive Scale Manual (2nd ed., SRS-2). Los Angeles, CA: Western Psychological Services; 2012.

15. Bolte S, Poustka F, Constantino JN. Assessing autistic traits: cross-cultural validation of the social responsiveness scale (SRS). Autism Res 2008;1:354-363.

16. Wigham S, McConachie H, Tandos J, Le Couteur AS; Gateshead Millennium Study core team. The reliability and validity of the Social Responsiveness Scale in a UK general child population. Res Dev Disabil 2012;33:944-950.

17. Tehrani-Doost M, Shahrivar Z, Torabi N, Ansari S, Haji-Esmaeelzadeh M, Saeed-Ahmadi S. Cross-cultural validation and normative data of the social responsiveness scale in a group of Iranian general child population. J Autism Dev Disord 2020;50:2389-2396.

18. Fombonne E, Marcin C, Bruno R, Tinoco CM, Marquez CD. Screening for autism in Mexico. Autism Res 2012;5:180-189.

19. Cen CQ, Liang YY, Chen QR, Chen KY, Deng HZ, Chen BY, et al. Investigating the validation of the Chinese Mandarin version of the Social Responsiveness Scale in a Mainland China child population. BMC Psychiatry 2017;17:51.

20. Gau SF, Liu LT, Wu YY, Chiu YN, Tsai WC. Psychometric properties of the Chinese version of the Social Responsiveness Scale. Res Autism Spectr Disord 2013;7:349-360.

21. Wang J, Lee LC, Chen YS, Hsu JW. Assessing autistic traits in a Taiwan preschool population: cross-cultural validation of the Social Responsiveness Scale (SRS). J Autism Dev Disord 2012;42:2450-2459.

22. Nguyen PH, Ocansey ME, Miller M, Le DTK, Schmidt RJ, Prado EL. The reliability and validity of the social responsiveness scale to measure autism symptomology in Vietnamese children. Autism Res 2019; 12:1706-1718.

23. Kamio Y, Moriwaki A, Inada N. Utility of teacher-report assessments of autistic severity in Japanese school children. Autism Res Treat 2013; 2013:373240.

24. Bolte S, Westerwald E, Holtmann M, Freitag C, Poustka F. Autistic traits and autism spectrum disorders: the clinical validity of two measures presuming a continuum of social communication skills. J Autism Dev Disord 2011;41:66-72.

25. Chan W, Smith LE, Hong J, Greenberg JS, Mailick MR. Validating the social responsiveness scale for adults with autism. Autism Res 2017; 10:1663-1671.

26. Takei R, Matsuo J, Takahashi H, Uchiyama T, Kunugi H, Kamio Y. 
Verification of the utility of the social responsiveness scale for adults in non-clinical and clinical adult populations in Japan. BMC Psychiatry 2014;14:302.

27. Stickley A, Tachibana Y, Hashimoto K, Haraguchi H, Miyake A, Morokuma S, et al. Assessment of autistic traits in children aged 2 to 4(1/2) years with the preschool version of the Social Responsiveness Scale (SRS-P): findings from Japan. Autism Res 2017;10:852-865.

28. Bong G, Kim JH, Hong Y, Yoon NH, Sunwoo H, Jang JY, et al. The feasibility and validity of autism spectrum disorder screening instrument: Behavior Development Screening for Toddlers (BeDevel)-A pilot study. Autism Res 2019;12:1112-1128.

29. Lord C, Rutter M, DiLavore P, Risi S, Gotham K, Bishop S. Autism Diagnostic Observation Schedule (ADOS-2) Modules 1-4. Los Angeles, CA: Western Psychological Services; 2012.

30. Yoo H, Bong G, Kwak Y, Lee M, Cho S, Kim B, et al. Korean Version of Autism Diagnostic Observation Schedule-Second Edition (ADOS-2). Seoul: Inpsyt; 2017.

31. Rutter M, LeCouteur A, Lord C. Autism Diagnostic Interviewed Revised ADI-R. Los Angeles, CA: Western Psychological Services; 2003.

32. World Health Organization. International Statistical Classification of Diseases and Related Health Problems 10th Revision Version for 2006. Geneva: World Health Organization; 1992.

33. Yoo H, Park K, Kwak Y, Cho S, Ban G, Kim B. Korean Version of Autism Diagnostic Interview-Revised (ADIR). Seoul: Inpsyt; 2007.

34. Schopler E, Reichler R, Rochen R. The Childhood Autism Rating Scale. Los Angeles, CA: Western Psychological Services; 1988.

35. Kim T, Park R. Korean Version of Childhood Autism Rating Scale. Seoul: Special Education; 1995.
36. Kwon HJ, Yoo HJ, Kim JH, Noh DH, Sunwoo HJ, Jeon YS, et al. Readjusting the cut-off score of the Korean version of the Childhood Autism Rating Scale for high-functioning individuals with autism spectrum disorder. Psychiatry Clin Neurosci 2017;71:725-732.

37. Rutter M, Bailey A, Lord C, Berument SK. Social Communication Questionnaire. Los Angeles, CA: Western Psychological Services; 2003.

38. Kim JH, Sunwoo HJ, Park SB, Noh DH, Jung YK, Cho IH, et al. A validation study of the Korean version of social communication questionnaire. J Korean Acad Child Adolesc Psychiatry 2015;26:197-208.

39. Pine E, Luby J, Abbacchi A, Constantino JN. Quantitative assessment of autistic symptomatology in preschoolers. Autism 2006;10:344-352.

40. Volkmar FR. Vineland Adaptive Behavior Scales, Second Edition. Encyclopedia of Autism Spectrum Disorders. New York: Springer; 2013.

41. Kim Y, Kim K, Yoon H, Kim W. Sequenced Language Scale for Infants (SELSI). Seoul: Special Education Publication Co.; 2003.

42. Oh M, Song DY, Bong G, Yoon NH, Kim SY, Kim JH, et al. Validating the autism diagnostic interview-revised in the Korean population. Psychiatry Investig 2021;18:196-204.

43. Camarata S. Early identification and early intervention in autism spectrum disorders: accurate and effective? Int J Speech Lang Pathol 2014; 16:1-10.

44. Warren Z, McPheeters ML, Sathe N, Foss-Feig JH, Glasser A, Veenstra-Vanderweele J. A systematic review of early intensive intervention for autism spectrum disorders. Pediatrics 2011;127:e1303-e1311.

45. Constantino JN. Deconstructing autism: from unitary syndrome to contributory developmental endophenotypes. Int Rev Psychiatry 2018;30: 18-24. 